$\mathrm{AB} 0942$

RADIOLOGICAL SACROILIITIS AFTER 18 YEARS OF FOLLOW-UP IN THE POPULATION-BASED NORDIC JUVENILE IDIOPATHIC ARTHRITIS (JIA) COHORT

Karin Qvarnström ${ }^{1}$, Mia Glerup ${ }^{2}$, Veronika Rypdal ${ }^{3}$, Ellen Dalen Arnstad ${ }^{4,5}$, Suvi Peltoniemi ${ }^{6}$, Maria Ekelund ${ }^{7}$, Anders Fasth ${ }^{8}$, Susan Nielsen ${ }^{9}$, Marek Zak ${ }^{9}$, Kristiina Aalto $^{10}$, Ellen Nordal ${ }^{3}$, Troels Herlin ${ }^{2}$, Marite Rygg ${ }^{4,11}$, Lillemor Berntson ${ }^{7}$, the Nordic Study Group of Pediatric Rheumatology (NoSPeR). ${ }^{1}$ Centre for Research and Development, Uppsala University/Region Gävleborg, Gävle, Sweden; ${ }^{2}$ Aarhus University Hospital, Department of Pediatrics, Aarhus, Denmark; ${ }^{3}$ Department of Pediatrics, University Hospital of North Norway, and Department of Clinical Medicine, UiT The Arctic University of Norway, Department of Clinical Medicine, Tromsø, Norway, ${ }^{4}$ NTNU - Norwegian University of Science and Technology, Department of Clinical and Molecular Medicine, Trondheim, Norway, ${ }^{5}$ Levanger Hospital, Nord-Trøndelag, Norway, ${ }^{6}$ University of Helsinki, Helsinki, Finland; 'Uppsala University, Department of Women's and Children's health, Uppsala, Sweden; ${ }^{8}$ Institute of Clinical Sciences, Sahlgrenska Academy, University of Gothenburg, Department of Pediatrics, Gothenburg, Sweden; ${ }^{9}$ Rigshospitalet Copenhagen University Hospital, Department of Pediatrics, Copenhagen, Denmark: ${ }^{10}$ University of Helsinki, Hospital for Children and Adolescents, Helsinki, Finland; ${ }^{11}$ St. Olavs Hospital, Trondheim, Norway

Background: A challenge with the present classification of JIA is the evolution of the disease over time. One category that is especially difficult to classify is enthesitis-related JIA (ERA).

Objectives: To longitudinally study radiologically diagnosed sacroilitis (SI) developed during the first 18 years in an aim to gain knowledge about classification challenges posed by the proposed, new classification (Martini A. et al. J Rheumatol. 2018 Oct; Epub ahead of print).

Methods: 510 consecutive cases of JIA with disease onset 1997 to 2000 were prospectively included in a Nordic, longitudinal, close to populationbased 18-year follow-up study, and $434(85 \%)$ had at least two follow-up visits during disease course. At the 18-year follow-up visit; 329 (76\%) attended a clinical visit, and $105(24 \%)$ a telephone interview. The follow-up period was $17.5 \pm 1.7$ years (mean \pm SD) after onset. Mean age of the study participants was $24.0 \pm 4.4$ years. Clinical data, collected at one, eight and 18 years after disease onset, were evaluated regarding variables for enthesitis/spondylitis-related arthritis compared to the other JIA categories. Results: In 376 participants evaluated for SI, radiology was performed on clinical suspicion, 26 (16 males, 10 females) developed radiologically verified sacroilitis (rad-SI) during the first 18 years of disease. Age at onset was significantly higher in this group compared to the other participants, median 9.9 (IQR 6.4-12.0) vs. 5.6 (IQR 2.6-9.5) years, ( $p=0.001$ ). Only 3/ 26 had rad-SI at eight-year follow-up.

Using the ILAR criteria 12/26 with rad-SI were classified as ERA after median 7 (IQR 6-10.2) months, $1 / 26$ as juvenile psoriatic arthritis, 5/26 as undifferentiated JIA because of psoriasis-related variables, the remaining had oligo- or polyarticular arthritis. At 18-year follow-up, 18/26 fulfilled criteria for ERA, 2/26 juvenile psoriatic arthritis, and 3/26 the undifferentiated category because of psoriasis-related variables, 3/26 had polyarticular RF negative or oligoarticular extended disease. Enthesitis, inflammatory back pain and $\mathrm{SI}$ joint tenderness were more common with rad-SI $(\mathrm{p}<0.001 ; \mathrm{p}<0.001 ; \mathrm{p}<0.001)$, also HLA-B27, $18 / 26$ vs. 62/351 $(\mathrm{p}<0.001)$, but not $1^{\text {st }}$ degree heredity for ankylosing spondylitis. Uveitis developed in 10/26 (38.5\%). Two participants developed IBD.

Conclusion: The majority of variables involved in the new proposed classification of enthesitis/spondylitis-related JIA were significantly more common during the first 18 years of disease in those that developed rad-SI. Psoriasis-related variables, no longer exclusion criteria for ERA, should be further evaluated as possible inclusion criteria, as well as higher age at onset.

Disclosure of Interests: Karin Qvarnström: None declared, Mia Glerup: None declared, Veronika Rypdal: None declared, Ellen Dalen Arnstad: None declared, Suvi Peltoniemi: None declared, Maria Ekelund: None declared, Anders Fasth: None declared, Susan Nielsen: None declared, Marek Zak: None declared, Kristiina Aalto: None declared, Ellen Nordal: None declared, Troels Herlin: None declared, Marite Rygg: None declared, Lillemor Berntson Consultant for: AbbVie, Speakers bureau: AbbVie

DOI: 10.1136/annrheumdis-2019-eular.4552

\section{AB0943 \\ CLINICAL AND LABORATORY PRESENTATION OF JUVENILE SJÖGREN'S SYNDROME IN A COHORT OF 30 PATIENTS}

Blanca Bica, Fernanda Zonis. UNIVERSIDADE FEDERAL DO RIO DE JANEIRO, INTERNAL MEDICINE, RIO DE JANEIRO, BraZil

Background: Sjögren's Syndrome (SS) is a chronic autoimmune disease, rare in childhood and adolescence, characterized mainly by xerostomia and xerophthalmia, which compromises exocrine glands causing inflammatory response that leads to glandular hyposecretion. SS is probably underdiagnosed in pediatric range due to differences in presentation regarding adults with this condition, causing low recognition of the disease in children.

Objectives: The authors describe demographic, clinical, laboratory and therapeutic profiles of a cohort of children with primary SS attended at a university center.

Methods: Retrospective analysis of 30 selected patients' medical records between 2005 and 2017 allowed collection of various data. Laboratory and additional investigations included documentation of ocular dryness (Schirmer test, Rose-Bengal stain); evidence of parotid involvement (scintiscan, sialometry); and histological evidence of lymphocytic infiltration of the minor salivary glands or other organs.

Results: 30 patients diagnosed with juvenile SS were selected: 22 girls $(73 \%)$ and 8 boys $(27 \%)$ with an average age of 11 years. The clinical characteristics were: parotid enlargement as the initial manifestation of the disease in 11 patients $(37 \%)$, and recurrent episode in 4 patients $(13,3 \%) .12$ patients $(40 \%)$ had xerostomia and 17 (57\%) xerophthalmia One patient $(3,3 \%)$ presented with leukocytoclastic vasculitis in lower limbs as first manifestation of the disease in association to recurrent parotid swelling. Two $(6,6 \%)$ patients presented neurological symptoms (1 peripheric sensory neuropathy and 1 with dysautonomic manifestations). Positive Schirmer test was observed in 11 patients (37\%), Rose Bengal stain in $10(33 \%)$. $76 \%$ of patients had abnormal salivary glands scintigraphy. In 8 patients $(30 \%)$ the salivary gland biopsy revealed compatible with Sjogren syndrome. 9 patients $(30 \%)$ presented positive RF, 14 patients $(46 \%)$ anti-Ro/SSA, 10 patients $(33 \%)$ anti-La/SSB, 28 patients $(93 \%)$ had +ve ANA. 50\% received glucocorticoid. Hydroxychloroquine was the drug most often used in 25 patients $(83 \%)$, followed by methotrexate in 12 patients $(40 \%)$, azathioprine in 4 patients $(13 \%)$ and cyclophosphamide in 3 patients $(10 \%)$. Only one patient required the use of human immunoglobulin and one leflunomide (3.3\%). Two patients $(6.6 \%)$ received rituximab.

Conclusion: The present study demonstrated the demographic, clinical aspects and laboratory and treatment in a series of patients with primary juvenile Sjögren's syndrome, a relatively rare condition, presenting an overview of this population in our hospital.

\section{REFERENCES}

[1] Primary Sjogren syndrome in the paediatric age: a multicentre survey. Rolando Cimaz, Annachiara Casadei, Carlos Rose, et al. Eur J Pediatr (2003) 162: 661-665.

[2] Primary Sjögren's syndrome in children and adolescents: Proposal for diagnostic criteria. J. Bartunková, A. Sedivá, J. Vencovsky, V. Tesar. Clinical and Experimental Rheumatology 1999; 17: 381-386

[3] Primary Sjo"gren syndrome in childhood: Report of a case and review of the literature. Nikolaos G. Nikitakis, Helen Rivera, Carmela Lariccia, John C. Papadimitriou, and John J. Sauk. Oral Surgery Oral Medicine Oral Pathology Radiol Endod 2003;96:42-7.

Disclosure of Interests: None declared

DOI: 10.1136/annrheumdis-2019-eular.6482

\section{AB0944 SJÖGREN'S SYNDROME IN CHILDREN: A CASE SERIES}

Masa Bizjak, Štefan Blazina, Tina Vesel Tajnšek, Tadej Avcin, Nataša Toplak. University Children's Hospital, University Medical Centre Ljubljana, Department of Allergology, Rheumatology and Clinical Immunology, Ljubljana, Slovenia

Background: The symptoms of pediatric Sjögren's syndrome (SS) are different than in adults. There are currently no validated pediatric diagnostic criteria or treatment guidelines for SS. In most cases adult criteria are used, but they apply poorly to children.

Objectives: To present pediatric patients with primary SS who were treated at University Children's Hospital (UCH) Ljubljana in the past 10 years.

Methods: Eight children with primary SS were identified. Demographic data, clinical and laboratory findings and therapy were analysed by retrospective review of medical records at UCH Ljubliana.

Results: Six girls and 2 boys were evaluated. The mean age at disease onset was 12.3 years (range 6.5 - 17) and mean age at diagnosis was 13.8 years (range 7.5 - 17.5). The mean follow-up duration was 2.8 years (range $0.5-8.5$ ). Four patients presented with recurrent bilateral parotitis, two with rash, one with arthralgia and fatigue and one with acute central nervous system vasculitis. The latter patient presented with rheumatic fever at the same time. During disease course arthritis and/or 
arthralgia was present in $5 / 8$, parotitis in $4 / 8$, oral symptoms in $4 / 8$, rash in $4 / 8$, fatigue in $3 / 8$ and ocular symptoms in $2 / 8$ patients. One patient developed calcinations on fingers. All patients had high titer of antinuclear antibodies, 7/8 were positive for anti-Ro and 5/8 for anti-La antibodies. Three patients were tested for rheumatoid factor and all were positive. 6/ 8 patients had elevated ESR and hypergammaglobulinemia. 4/8 patients had elevated serum amylase.

Biopsy of salivary glands was performed in 5 patients and foci of lymphocytic infiltration were shown in all of them. Further two patients had salivary gland changes on MRI and/or US. On ophthalmologic evaluation 3 patients had positive Schirmer test and one had unstable tear film. One patient had CNS vasculitis and two decreased diffusing capacity of the lungs. Other patients showed no signs of internal organ involvement. $4 / 8$ patients were treated with NSAIDs, $7 / 8$ patients with hydroxychloroquine, 2/8 also received corticosteroids and one patient MMF. At this point the patient with CNS vasculitis has stable changes on head MRI without clinical symptoms. Other patients have no signs of internal organ damage. Calcinations that appeared on fingers in one patient are not progressing.

Conclusion: Most common symptoms in our cohort were arthritis and/or arthralgia, parotitis, oral symptoms and rash. Half of the children presented with recurrent parotitis. One child developed calcinations on fingers, which have not yet been described in patients with SS. Pediatric SS differs from adult SS and specific pediatric diagnostic criteria would improve management of these patients.

\section{REFERENCES}

[1] Yokogawa N, Lieberman SM, Sherry DD, Vivino FB. Features of childhood Sjogren's syndrome in comparison to adult Sjogren's syndrome: considerations in establishing child-specific diagnostic criteria. Clin Exp Rheumatol 2017; 34(2): 343-351.

Disclosure of Interests: None declared

DOI: 10.1136/annrheumdis-2019-eular.3817

\section{AB0945 CO-MORBID AFFECTIONS FORMATION IN CHILDREN WITH SYSTEMIC LUPUS ERYTHEMATOSUS}

Ljudmila Bohmat $^{1,2}$, Natalia Shevchenko ${ }^{1,3}$, Irina Bessonova ${ }^{1,2} .{ }^{1}$ Institute of Children and Adolescents Health Care, Department of cardiorheumatology, Kharkiv, Ukraine; ${ }^{2}$ V.N. Karazin National University, Department of pediatrics, Kharkiv, Ukraine; ${ }^{3}$ V.N. Karazin National University, Department of pediatrics № 2 , Kharkiv, Ukraine

Background: In children with systemic lupus erythematosus (SLE) due to the cascade of immune-inflammatory reactions there is a development of systemic vascular endothelium lesion, which causes not only clinical manifestations of the main process, but also leads to the damage of vital organs and systems, the development of metabolic disorders. Extensive damage aggravates more the course of the disease, worsens its prognosis, complicates the response to therapy and reduces the quality of life of patients.

Objectives: The aim of study was to determine the frequency and variants of comorbid conditions in children depending on the duration and activity of the disease and feathers of the therapeutic complex.

Methods: A survey was conducted on 41 patients with SLE: 37 girls $(90.2 \%), 4$ boys $(9.8 \%)$; the duration of the disease is up to 3 years in 20 patients (48.8\%)and more than 3 years in 21 (51,2\%). Presence of comorbid conditions determinates as the cardiovascular system, kidney, liver and lungs functions changes. General clinical trials included autoantibodies, disease activity and drugs assessment. Total cholesterol, triglycerides, high density lipoprotein cholesterol, low-density lipoprotein cholesterol, apolipoprotein B, ApoA-I and lipoprotein- $\alpha$ were evaluated. The state of the blood coagulation system was also studied: fibrinogen of the blood, prothrombin index, thrombin time, active partial thrombin time, D-dimer, international normalized ratio. Bone mineral density was determined.

Results: In $74.3 \%$ of children with SLE, the presence of comorbid conditions characterized by pathology of the cardiovascular system, kidneys, liver, the organ of vision, pulmonary system is established. Besides, atherogenic dislipoproteinemia has been detected in 60,0\%, disorders in the hemostasis system were in $25,0 \%$ of patients and $43,0 \%$ persons have osteopenia. Children with SLE and the presence of comorbid conditions, especially with the involvement of the liver and kidneys and the longterm preservation of the disease activity (SRP, ANA, anti-DNA), are more likely to develop atherogenic dislipoproteinemia and hypercoagulation (increase in the prothrombin index, fibrinogen and D- dimer). The values of the comorbidity index in patients with SLE increased while maintaining the activity of the process and using high doses of glucocorticosteroids (GCS) including pulse therapy. Comorbidity index was $2.9 \pm 0.5$ points in the patients who received the GCS pulse therapy; $2.5 \pm 0.3$ in the cases of treatment by combination GCS + azathioprine; 2,0 $\pm 0,6$ in the children without GCS; $\mathrm{p}<0,05)$. ANA-positivity was also accompanied by higher values of the comorbidity index $(2.8 \pm 0.3$ vs. $1.4 \pm 0.5, p<0.05)$. Conclusion: Children with SLE in the long-term course of the disease and while maintaining the activity of the pathologic process (ANA, DNA antibodies high level) have a formation of lesions of systems and organs, the lipids metabolism disorders, prolonged hypercoagulation and the development of osteopenia. Aggressive therapy for reducing the activity of the autoimmune process helps to prevent the formation of comorbid states and persistent metabolic disorders.

Disclosure of Interests: None declared

DOI: 10.1136/annrheumdis-2019-eular.3858

\section{AB0946 QUALITY IMPROVEMENT IN PEDIATRIC RHEUMATOLOGY: A NEW APPROACH TO CARE IN QATAR}

Sharon Bout-Tabaku. Sidra Medicine, Pediatric Rheumatology, Doha, Qatar

Background: Quality improvement (QI) projects have been established in many pediatric and adult rheumatology centers around the world to enable real time improvement of health delivery and outcomes. QI has been driven by the Institute of Medicine, patients, professional organizations and the providers. Notably, another driver is the discrepancy between the availability of better and targeted therapies and poor disease outcomes. QI allows for healthcare teams to decide on improvement goals to address gaps in care and implement these goals in sustainable ways, evaluate the impact and start a new cycle of improvement. In so doing teams collaborate, learn from each other, and improve variability in a short time frame. In small practices with limited resources, effective QI projects allows for understanding the gaps in the processes, data collec tion and several process improvements to be done by different team members, in short cycles that allow for real time data evaluation and fine tuning processes.

Objectives: We describe the chosen quality measures, implementation of processes and first 6 months of data at a newly opened Children's Hospital in Doha, Qatar.

Methods: We chose quality measures that have been proposed in the lit erature and have been implemented in large learning collaboratives. We worked with the informatics team to build measures that are accu rately captured and retrievable from the electronic medical record. Additionally we worked with our Ophthalmology clinic to ensure a smooth referral pathway that is coordinated by nursing staff. Our EMR measures were 1) the screening for drug toxicity among patients receiving methotrexate or leflunomide within 3-4 month of receiving Methotrexate 2) the time between referral to and visit in Ophthalmology, to screen for toxicity among patients receiving hydroxychloroquine 3) time between referral to and visit in Ophthalmology, to screen for uveitis in children diagnosed with juvenile idiopathic arthritis.

Our nursing teams developed a manual joint injection log 1) to capture whether the time between referral for procedure and procedure date occurred within 2 weeks, and monitor side effects.

We examined data between June to December 2018 from the pediatric rheumatology patient population of 420 unique patients.

Results: $96 \%$ of children receiving methotrexate or leflunomide were screened for methotrexate toxicity within 3-4 months of the medicine being dispensed.

$50 \%$ of children receiving hydroxychloroquine were seen by Ophthalmology within 4 months of the referral.

$71 \%$ of children with JIA were seen by Ophthalmology within 1 month of the referral.

$100 \%$ of children had joint injections performed within 14 days of the referral.

Conclusion: We described the development, implementation and results of our pediatric rheumatology QI projects. To our knowledge it is the firs of a kind in Qatar and in the area. For our growing practice of 600 patient visits our data shows areas of great performance and poor performance, specifically in eye care for our patients. Barriers included education of teams about QI measures and processes, collaborating with other services on negotiating best patient practices.

Successful implementation in our processes underlines the need for further data collection and additional improvement cycles. Additionally, in resource poor areas, it is essential to make good use of the EMR and 